\title{
Optimum Power Allocation for Average Power Constrained Jammers in the Presence of Non-Gaussian Noise
}

\author{
Suat Bayram, N. Denizcan Vanli, Berkan Dulek, Ilhan Sezer, and Sinan Gezici, Senior Member, IEEE
}

\begin{abstract}
We study the problem of determining the optimum power allocation policy for an average power constrained jammer operating over an arbitrary additive noise channel, where the aim is to minimize the detection probability of an instantaneously and fully adaptive receiver employing the Neyman-Pearson (NP) criterion. We show that the optimum jamming performance can be achieved via power randomization between at most two different power levels. We also provide sufficient conditions for the improvability and nonimprovability of the jamming performance via power randomization in comparison to a fixed power jamming scheme. Numerical examples are presented to illustrate theoretical results.
\end{abstract}

Index Terms-Jammer, randomization, detection, false-alarm, Neyman-Pearson, radar.

\section{INTRODUCTION}

$\mathbf{R}$ ECENTLY, signal and power randomization approaches have received considerable interest in the literature [1][5]. Several papers have addressed different aspects of the jammer power randomization/allocation problem. The convexity properties of the error probability with respect to jammer power is investigated in [4] under the antipodal signaling scheme by restricting the analysis to the class of symmetric unimodal jammer noise probability density functions (PDFs) and to maximum likelihood (ML) receivers. It is shown that, when the error probability as a function of the jammer power has a single point of inflection, there exists a critical value $\gamma_{c}$ for the average jammer power below which the optimum strategy reduces to on-off time-sharing with $\gamma_{c}$ as its on-power. On the other hand, jamming at the maximum available power limit is shown to be more advantageous beyond that critical value. This discussion is extended from binary modulations to arbitrary signal constellations in [5] by concentrating on the ML detection case in an additive white Gaussian noise (AWGN) channel. It is stated that the symbol error rate (SER) performance of the target receiver can be degraded via appropriate power/time sharing under a fixed average jammer noise power. Also, the authors show that two power levels are sufficient to achieve optimum performance under moderate and high signal-to-noise ratio (SNR) conditions while jamming at the average power limit is the best strategy at low SNRs due to plenty of jamming power.

In [6], the problem of optimal noise jamming of radar signals is addressed by devising a game between a smart jammer operating under a power constraint and a decision maker (DM) that is fully aware of jammer's actions. It is

Manuscript received January 13, 2012. The associate editor coordinating the review of this letter and approving it for publication was J. Choi.

S. Bayram is with the Dept. of Electrical and Computer Engineering, The Ohio State University, Columbus, OH 43210, USA.

I. Sezer is with the Dept. of Economics, Princeton University, Fisher Hall, Princeton, NJ 08544, USA.

N. D. Vanli, B. Dulek, and S. Gezici are with the Dept. of Electrical and Electronics Eng., Bilkent University, Bilkent, Ankara 06800, Turkey (e-mail: gezici@ee.bilkent.edu.tr).

Digital Object Identifier 10.1109/LCOMM.2012.052112.120098 shown that the optimal PDF for the additive narrowband jamming waveform approaches Gaussian as the sample number increases when the DM employs the Neyman-Pearson (NP) criterion to detect the slowly fading narrowband target echo. A similar game is formulated in [7] where one party tries to minimize the SNR at the output of the radar receiver through the appropriate design of the spectral density of the jamming noise while the other aims to maximize it by selecting a filter function. In [8], the intentional use of jamming transmission is discussed as a remedy to ensure the physical layer security against eavesdropper nodes in wireless networks with power constraints. Communications in the presence of jamming is considered in [9] from an information theoretic point of view. It is shown that optimum minimax strategies result in Gaussian input and Gaussian jamming when both coder and jammer are subject to average power constraints. In [10], a game-theoretic framework is established to discuss the effects of jamming on the channel capacity by modeling the communicator and the jammer as the players of a two-person zero-sum game subject to certain constraints.

Although various aspects of the jamming problem have been investigated in [6]-[10] and the optimum jammer power allocation problem has been studied for Gaussian PDFs and ML receivers in [4], [5], no studies have considered the case of arbitrary jammer and background noise PDFs and generic decision rules at the receivers. In this letter, we study the problem of determining the optimum power allocation policy for an average power constrained jammer operating over an arbitrary additive noise channel, where the aim is to minimize the detection probability of an instantaneously and fully adaptive receiver employing generic decision rules in the NP framework. In addition, both the jammer and the receiver are assumed to operate ideally in the sense that the receiver is able to update its decision rule instantaneously for any observed jammer and background noise statistics, and likewise the jammer is fully informed of the signal and noise distributions at the receiver. Therefore, the proposed study can also be considered to provide theoretical limits on the performance of a jammer against a smart receiver. Under the stated conditions, we show that the optimum power allocation policy for an average power constrained jammer involves randomization between at most two different power levels. We also provide conditions for the improvability and nonimprovability of the jamming performance via power randomization with respect to a fixed power jamming scheme.

\section{Analysis of Optimum Power Allocation FOR AVERAge Power Constrained JAMMERS}

Consider a receiver that aims to detect a target signal in the presence of jamming. In other words, the receiver performs a decision between two hypotheses corresponding to the absence and presence of the target signal. The observation vector at the 
receiver is expressed as

$$
\mathcal{H}_{0}: \mathbf{y}=\gamma \mathbf{n}+\boldsymbol{\epsilon}, \quad \mathcal{H}_{1}: \mathbf{y}=\mathbf{s}+\gamma \mathbf{n}+\boldsymbol{\epsilon},
$$

where $\mathcal{H}_{0}$ and $\mathcal{H}_{1}$ denote the null and alternative hypotheses, respectively, $\mathbf{y} \in \mathbb{R}^{K}$ is the observation vector, target signal $\mathbf{s}$ is modeled as a random vector with $\operatorname{PDF} p_{\mathbf{s}}(\cdot), \boldsymbol{\epsilon}$ is the background noise with PDF $p_{\boldsymbol{\epsilon}}(\cdot)$, and $\mathbf{n}$ denotes the normalized noise of the jammer with PDF $p_{\mathbf{n}}(\cdot)$, which is known by the receiver. The power of the jammer is allocated over time through the scalar variable $\gamma$ with the PDF $p_{\gamma}(\cdot)$. Considering a smart receiver, it is assumed that the value of $\gamma$ is learned instantly by the receiver [6]. For convenience, we assume that $\operatorname{trace}\{\operatorname{Cov}(\mathbf{n})\}=1$, and the average power constraint on the jammer is represented by

$$
\mathrm{E}_{\gamma}\left\{\gamma^{2}\right\} \leq \beta
$$

where $\beta$ denotes the average power limit. In other words, the jammer can time/power share subject to the constraint in (2).

The receiver operates in the NP framework and achieves a certain detection probability under the false alarm constraint $\alpha$. The decision rule at the receiver is modeled as a generic one, denoted by $\phi_{\gamma}(\mathbf{y})$, which corresponds to the probability of selecting $\mathcal{H}_{1}$. The aim of the jammer is to minimize the average detection probability of the receiver under the average power constraint on the jammer noise by a suitable allocation of the jammer power as determined by the probability distribution of $\gamma$. Since the receiver is modeled as a smart one, it knows the instantaneous value of $\gamma$ and adapts itself according to the allocation policy of the jammer immediately. This is the main reason why the decision rule is modeled as a function of $\gamma$, that is, $\phi_{\gamma}(\cdot)$. In other words, for each value of $\gamma$, the receiver can design a corresponding decision rule. The formulation is kept quite generic by assuming that the designed decision rules can be optimal or suboptimal as long as the false alarm constraint is satisfied at the receiver, that is, each decision rule sets the probability of false alarm at the receiver to $\alpha$.

The proposed formulation in this study provides guidelines for system design by presenting theoretical limits on the performance of jamming. Specifically, the best jamming performance is calculated against a smart receiver considering generic decision rules and noise PDFs. On the one hand, the smart receiver assumption results in the worst-case scenario for the jammer since the receiver knows the PDF of jammer noise $\mathbf{n}$, can instantly learn the policy of the jammer, and adapt itself accordingly. In the absence of this assumption, more efficient jamming can be performed. On the other hand, if the jammer does not employ the optimal jamming strategy developed in this study due to some practical considerations, then inferior jamming performance will be observed. All in all, the proposed study provides both lower and upper bounds on the jamming performance.

In order to provide a mathematical formulation of the problem, the probability of detection is determined first:

$$
\mathrm{P}_{\mathrm{D}}(\gamma)=\int_{\mathbb{R}^{K}} \phi_{\gamma}(\mathbf{y}) p_{1}(\mathbf{y}) d \mathbf{y},
$$

where $p_{1}(\mathbf{y})$ is the PDF of the observation under the alternative hypothesis. Assuming that $\mathbf{n}, \boldsymbol{\epsilon}$ and $\mathbf{s}$ are independent, $p_{1}(\mathbf{y})$ can be expressed as

$$
p_{1}(\mathbf{y})=\int_{\mathbb{R}^{K}} \int_{\mathbb{R}^{K}} \frac{1}{|\gamma|} p_{\mathbf{n}}\left(\frac{\mathbf{y}-\mathbf{s}-\mathbf{e}}{\gamma}\right) p_{\boldsymbol{\epsilon}}(\mathbf{e}) p_{\mathbf{s}}(\mathbf{s}) d \mathbf{e} d \mathbf{s} .
$$

Thus, $\mathrm{P}_{\mathrm{D}}(\gamma)$ in (3) becomes

$$
\begin{aligned}
\mathrm{P}_{\mathrm{D}}(\gamma)= & \int_{\mathbb{R}^{K}} \phi_{\gamma}(\mathbf{y}) \int_{\mathbb{R}^{K}} \int_{\mathbb{R}^{K}} \frac{1}{|\gamma|} \\
& \cdot p_{\mathbf{n}}\left(\frac{\mathbf{y}-\mathbf{s}-\mathbf{e}}{\gamma}\right) p_{\boldsymbol{\epsilon}}(\mathbf{e}) p_{\mathbf{s}}(\mathbf{s}) d \mathbf{e} d \mathbf{s} d \mathbf{y} .
\end{aligned}
$$

The average probability of detection can be calculated from the expectation of $\mathrm{P}_{\mathrm{D}}(\gamma)$ as follows:

$$
\mathrm{P}_{\mathrm{D}}^{\mathrm{avg}}=\int_{-\infty}^{\infty} p_{\gamma}(\gamma) \mathrm{P}_{\mathrm{D}}(\gamma) d \gamma=\mathrm{E}_{\gamma}\left\{\mathrm{P}_{\mathrm{D}}(\gamma)\right\}
$$

where $\mathrm{P}_{\mathrm{D}}(\gamma)$ is as in (5).

The aim is to find the optimum power allocation policy, i.e., $p_{\gamma}(\cdot)$, for the jammer which minimizes the average detection probability of the receiver under the average power constraint on the jammer. Based on (2) and (6), the problem can be formulated as follows:

$$
\underset{p_{\gamma}(\cdot)}{\operatorname{minimize}} \mathrm{E}_{\gamma}\left\{\mathrm{P}_{\mathrm{D}}(\gamma)\right\} \text { subject to } \mathrm{E}_{\gamma}\left\{\gamma^{2}\right\} \leq \beta
$$

In the literature, optimization problems that are in similar forms have been investigated in various studies such as [1]-[3]. A similar approach is used to obtain the following proposition:

Proposition 1: Assume that $\gamma$ belongs to a finite closed set and $\mathrm{P}_{\mathrm{D}}(\gamma)$ in (5) is a continuous function. Then, the PDF for an optimum power allocation policy can be expressed as

$$
p_{\gamma}(\gamma)=\lambda \delta\left(\gamma-\gamma_{1}\right)+(1-\lambda) \delta\left(\gamma-\gamma_{2}\right), \quad \lambda \in[0,1]
$$

Proof: A similar approach to those in [1], [2] can be employed in this proof. Define sets $U$ and $W$ as $U=\left\{\left(u_{1}, u_{2}\right)\right.$ : $\left.u_{1}=\gamma^{2}, u_{2}=\mathrm{P}_{\mathrm{D}}(\gamma), \forall \gamma \in\left[\gamma_{\min }, \gamma_{\max }\right]\right\}$ and $W=$ $\left\{\left(w_{1}, w_{2}\right): w_{1}=\mathrm{E}_{\gamma}\left\{\gamma^{2}\right\}, w_{2}=\mathrm{E}_{\gamma}\left\{\mathrm{P}_{\mathrm{D}}(\gamma)\right\}, \forall p_{\gamma}(\cdot)\right\}$, where $\gamma_{\min }$ and $\gamma_{\max }$ denote the minimum and maximum possible values of $\gamma$, respectively. As in [1], [2], it can be shown that the convex hull of $U$ is equal to $W$. Then, from Carathéodory's theorem [11], it is deduced that any point in $W$ can be expressed as a convex combination of at most three points in $U$. In addition, since an optimal PDF should achieve the minimum value, the solution should stay on the boundary of $W$, resulting in a convex combination of at most two points in $U$. (Due to the assumptions in the proposition, $W$ is a closed set; hence, it contains its boundary [1].) Therefore, an optimal solution can be expressed as given in (8).

Based on Proposition 1, the optimal jamming policy can be obtained from a simplified version of (7) as follows:

$$
\begin{array}{ll}
\underset{\lambda, \gamma_{1}, \gamma_{2}}{\operatorname{minimize}} & \lambda \mathrm{P}_{\mathrm{D}}\left(\gamma_{1}\right)+(1-\lambda) \mathrm{P}_{\mathrm{D}}\left(\gamma_{2}\right) \\
\text { subject to } & \lambda \gamma_{1}^{2}+(1-\lambda) \gamma_{2}^{2} \leq \beta, \quad \lambda \in[0,1] .
\end{array}
$$

In this case, instead of searching over all possible PDFs, a search over only three variables is sufficient to obtain the optimal solution.

In order to evaluate the jamming performance of the optimum power allocation algorithm specified by (9), comparisons can be performed against the deterministic jamming approach in which the jammer transmits at the maximum power limit all the time; that is, $p_{\gamma}(\gamma)=\delta(\gamma-\sqrt{\beta})$. Then it becomes im- 
portant to specify, without solving the optimization problem in (9), whether power randomization can provide improvements over deterministic jamming. For that purpose, a sufficient condition for improvability, similar to those in [1], [2], is presented first.

Proposition 2: Assume $\gamma$ is a nonnegative variable 1 and define function $\mathrm{F}(a)$ as $\mathrm{F}(a) \triangleq \mathrm{P}_{\mathrm{D}}(\sqrt{a})$. If $\mathrm{F}(a)$ is strictly concave at $a=\beta$, meaning that $\mathrm{F}^{\prime \prime}(\beta)<0$ when $\mathrm{F}(a)$ is second-order continuously differentiable around $\beta$, then power randomization improves the jamming performance over the deterministic approach.

Proof: Under the conditions in the proposition, the concavity of $\mathrm{F}$ implies that there exists infinitesimally small $\nu$ such that $0.5 \mathrm{~F}(\beta+\nu)+0.5 \mathrm{~F}(\beta-\nu)<\mathrm{F}(\beta)$. Consider a power randomization approach specified by $p_{\gamma}(\gamma)=0.5 \delta(\gamma-$ $\sqrt{\beta+\nu})+0.5 \delta(\gamma-\sqrt{\beta-\nu})$. Then, the average detection probability becomes $\mathrm{E}_{\gamma}\left\{\mathrm{P}_{\mathrm{D}}(\gamma)\right\}=0.5 \mathrm{P}_{\mathrm{D}}(\sqrt{\beta+\nu})+$ $0.5 \mathrm{P}_{\mathrm{D}}(\sqrt{\beta-\nu})=0.5 \mathrm{~F}(\beta+\nu)+0.5 \mathrm{~F}(\beta-\nu)<\mathrm{F}(\beta)=$ $\mathrm{P}_{\mathrm{D}}(\sqrt{\beta})$. In other words, via power randomization, a lower average detection probability than that of the deterministic approach, $\mathrm{P}_{\mathrm{D}}(\sqrt{\beta})$, is achieved. In addition, the power randomization approach satisfies the average power constraint since $\mathrm{E}_{\gamma}\left\{\gamma^{2}\right\}=0.5(\sqrt{\beta+\nu})^{2}+0.5(\sqrt{\beta-\nu})^{2}=\beta$. Hence, a lower detection probability (i.e., improved jamming performance) is achieved via power randomization under the average power constraint. Therefore, the conditions in the proposition are sufficient conditions under which the jamming performance of the deterministic approach can be improved.

Next, a necessary and sufficient condition for nonimprovability is obtained.

Proposition 3: Let $\gamma$ take values in $\left[0, \gamma_{\max }\right]$, where $\gamma_{\max }$ denotes the maximum possible value of $\gamma$. Then power randomization cannot improve jamming performance over the deterministic approach if and only if there exist $\theta \leq 0$ such that

$$
\mathrm{P}_{\mathrm{D}}(\sqrt{a}) \geq(a-\beta) \theta+\mathrm{P}_{\mathrm{D}}(\sqrt{\beta}), \quad \forall a \in\left[0, \gamma_{\max }^{2}\right]
$$

Proof: In order to prove the sufficiency of the condition in the proposition for nonimprovability, consider a generic power randomization policy specified by $p_{\gamma}(\gamma)=\lambda \delta(\gamma-$ $\left.\gamma_{1}\right)+(1-\lambda) \delta\left(\gamma-\gamma_{2}\right)$. Then, the aim is to prove that, under the conditions in the proposition, the detection probability in the deterministic case, $\mathrm{P}_{\mathrm{D}}(\sqrt{\beta})$, cannot be reduced via power randomization; that is, defining $a_{1} \triangleq \gamma_{1}^{2}$ and $a_{2} \triangleq \gamma_{2}^{2}$, $\lambda \mathrm{P}_{\mathrm{D}}\left(\sqrt{a_{1}}\right)+(1-\lambda) \mathrm{P}_{\mathrm{D}}\left(\sqrt{a_{2}}\right) \geq \mathrm{P}_{\mathrm{D}}(\sqrt{\beta})$ for all $a_{1}, a_{2} \in$ $\left[0, \gamma_{\text {max }}^{2}\right]$ that satisfy $\lambda a_{1}+(1-\lambda) a_{2} \leq \beta$. From (10), the following inequality can be obtained:

$$
\begin{aligned}
\lambda \mathrm{P}_{\mathrm{D}}\left(\sqrt{a_{1}}\right)+(1-\lambda) \mathrm{P}_{\mathrm{D}}\left(\sqrt{a_{2}}\right) \geq \\
\mathrm{P}_{\mathrm{D}}(\sqrt{\beta})-\theta\left(\beta-\left(\lambda a_{1}+(1-\lambda) a_{2}\right)\right)
\end{aligned}
$$

for all $a_{1}, a_{2} \in\left[0, \gamma_{\max }^{2}\right]$. Since $\theta \leq 0$ and $\lambda a_{1}+(1-\lambda) a_{2} \leq \beta$ due to the average power constraint, the expression in the second line of (11) is always larger than or equal to $\mathrm{P}_{\mathrm{D}}(\sqrt{\beta})$. Hence, $\lambda \mathrm{P}_{\mathrm{D}}\left(\sqrt{a_{1}}\right)+(1-\lambda) \mathrm{P}_{\mathrm{D}}\left(\sqrt{a_{2}}\right) \geq \mathrm{P}_{\mathrm{D}}(\sqrt{\beta})$.

The necessity part of the proof is not presented due to the space limitation.

Remark 1: If $\mathrm{P}_{\mathrm{D}}(\sqrt{a})$ is first-order continuously differentiable, the only possibility for $\theta$ in Proposition 3 is the slope of the tangent line to $\mathrm{P}_{\mathrm{D}}(\sqrt{a})$ at $a=\beta$, that is, $\theta=$

\footnotetext{
${ }^{1}$ Similar arguments can also be provided for negative values of $\gamma$.
}

$\mathrm{P}_{\mathrm{D}}^{\prime}(\sqrt{\beta}) /(2 \sqrt{\beta})$. It is also noted that $\mathrm{P}_{\mathrm{D}}^{\prime}(\sqrt{\beta}) \leq 0$ is a necessary condition for nonimprovability because if $\mathrm{P}_{\mathrm{D}}^{\prime}(\sqrt{\beta})>0$, the jamming performance of the deterministic approach can simply be improved by employing $p_{\gamma}(\gamma)=\delta(\gamma-\sqrt{\beta}+\zeta)$ where $\zeta$ is an infinitesimally small positive number.

\section{NumERICAL RESUlTS AND CONCLUSIONS}

In this section, a numerical example is presented for scalar observations modeled as in (1). In the example, $s$ is assumed to be a positive constant, and jammer's standard noise $n$ and the background noise $\epsilon$ are modeled as symmetric Gaussian mixture noise. ${ }^{2}$ Specifically, the PDFs of $n$ and $\epsilon$ are given by

$$
p_{\epsilon}(\epsilon)=\sum_{i=1}^{M} \omega_{i} \psi_{i}\left(\epsilon-\mu_{i}\right), p_{\mathrm{n}}(\mathrm{n})=\sum_{i=1}^{\tilde{M}} \tilde{\omega}_{i} \tilde{\psi}_{i}\left(\mathrm{n}-\tilde{\mu}_{i}\right)
$$

where $M$ and $\tilde{M}$ denote the number of Gaussian components in the mixture noise PDFs, $\mu_{i}$ and $\tilde{\mu}_{i}$ are the mean values of the Gaussian components, $\sum_{i=1}^{M} \omega_{i}=\sum_{i=1}^{M} \tilde{\omega}_{i}=1, \omega_{i} \geq$ $0, \tilde{\omega}_{i} \geq 0, \psi_{i}(\mathrm{y})=\left(1 / \sqrt{2 \pi} \sigma_{i}\right) \exp \left\{-\mathrm{y}^{2} /\left(2 \sigma_{i}^{2}\right)\right\}$ for $i=$ $1, \ldots, M$, and $\tilde{\psi}_{i}(\mathrm{y})=\left(1 / \sqrt{2 \pi} \tilde{\sigma}_{i}\right) \exp \left\{-\mathrm{y}^{2} /\left(2 \tilde{\sigma}_{i}^{2}\right)\right\}$ for $i=$ $1, \ldots, \tilde{M}$, with $\sigma_{i}$ and $\tilde{\sigma}_{i}$ denoting the standard deviations of the Gaussian components in the mixture noise. The parameters are assumed to satisfy the necessary constraints so that the PDFs are symmetric around the origin.

A single threshold decision rule is employed at the receiver which sets its threshold value accordingly to maintain a constant false alarm rate of $\alpha$ as $\gamma$ varies. Specifically, the decision rule at the receiver is given by

$$
\phi_{\gamma}(\mathrm{y})=\left\{\begin{array}{ll}
1, & \mathrm{y}>\eta_{\gamma} \\
0, & \mathrm{y} \leq \eta_{\gamma}
\end{array},\right.
$$

where $\eta_{\gamma}$ is the threshold value that is selected to equate the probability of false alarm to $\alpha$. Based on (1), (12), and (13), the probability of false alarm expression can be obtained and threshold $\eta_{\gamma}$ can be calculated as follows:

$$
\mathrm{P}_{\mathrm{F}}(\gamma)=\sum_{i=1}^{M} \sum_{j=1}^{\tilde{M}} \omega_{i} \tilde{\omega}_{j} Q\left(\frac{\eta_{\gamma}-\mu_{i}-\gamma \tilde{\mu}_{j}}{\sqrt{\sigma_{i}^{2}+\gamma^{2} \tilde{\sigma}_{j}^{2}}}\right)=\alpha,
$$

where $Q(x)=(1 / \sqrt{2 \pi}) \int_{x}^{\infty} \mathrm{e}^{-t^{2} / 2} d t$ denotes the $Q$-function. Similarly, the resulting probability of detection is expressed as

$$
\mathrm{P}_{\mathrm{D}}(\gamma)=\sum_{i=1}^{M} \sum_{j=1}^{\tilde{M}} \omega_{i} \tilde{\omega}_{j} Q\left(\frac{\eta_{\gamma}-\mathrm{s}-\mu_{i}-\gamma \tilde{\mu}_{j}}{\sqrt{\sigma_{i}^{2}+\gamma^{2} \tilde{\sigma}_{j}^{2}}}\right),
$$

where $\eta_{\gamma}$ is substituted from (14).

In the simulations, the PDF of the background noise $\epsilon$ in (12) is specified by $M=2, \omega_{1}=\omega_{2}=0.5, \mu_{1}=2$, $\mu_{2}=-2$, and $\sigma_{1}=\sigma_{2}=0.28$. For the PDF of jammer's standard noise n, two different scenarios are considered: The first scenario is the Gaussian jammer case in which $\mathrm{n}$ is modeled as a zero mean Gaussian random variable with unit variance (i.e., $\tilde{M}=1, \tilde{\omega}_{1}=1, \tilde{\mu}_{1}=0$, and $\tilde{\sigma}_{1}=1$ in (12)). The second scenario is the non-Gaussian jammer case in which the PDF of $\mathrm{n}$ is modeled as in (12) with $\tilde{M}=2$, $\tilde{\omega}_{1}=0.1, \tilde{\omega}_{2}=0.9, \tilde{\mu}_{1}=\tilde{\mu}_{2}=0, \tilde{\sigma}_{1}=3$, and $\tilde{\sigma}_{2}=1 / 3$. In this scenario, jammer's standard noise is again a zero mean

\footnotetext{
${ }^{2}$ Due to symmetry, only nonnegative values of $\gamma$ are considered in the simulations.
} 


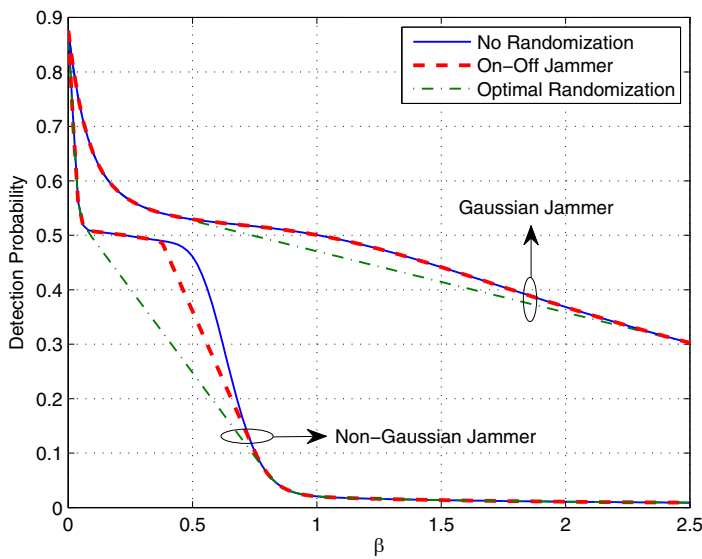

Fig. 1. Detection probability vs. average power constraint level $\beta$ for optimal power randomization, on-off jammer, and no randomization approaches.

and unit variance random variable, but it has heavier tails than a Gaussian random variable [12]. In addition, $\mathrm{s}=5$ and $\alpha=0.001$ are used.

In Fig. 1, the detection probabilities are plotted versus the average power constraint level $\beta$ for both the Gaussian and non-Gaussian jammer scenarios. The proposed optimum power allocation strategy obtained from (9) ("Optimal Randomization"), the deterministic strategy that sets $\gamma=\sqrt{\beta}$ ("No Randomization"), and the on-off jamming strategy ("On-Off Jammer") are considered. For the on-off jamming strategy, the jammer is on with probability $\lambda_{\text {on }}$ transmitting at a power level of $\gamma_{\text {on }}$, and it is off with probability $\left(1-\lambda_{\text {on }}\right)$ [4]. In addition, $\lambda_{\text {on }}=\beta / \gamma_{\text {on }}^{2}$ so that the power constraint is satisfied with equality. The optimal values of $\lambda_{\text {on }}$ and $\gamma_{\text {on }}$ that minimize the detection probability are calculated based on a one-dimensional search due to the relation between $\lambda_{\text {on }}$ and $\gamma_{\text {on. }}$. From Fig. 1, it is observed that the proposed optimum power allocation strategy results in the most effective jamming, leading to lower detection probabilities at the receiver, for intermediate values of $\beta$. However, for large and small values of $\beta$, the optimum power allocation does not provide (significant) improvements over the other approaches. In addition, the on-off jamming strategy can provide improvements over the no randomization case in the non-Gaussian jammer scenario. However, it is not optimal in general since it cannot always achieve the detection probabilities of the optimum power allocation strategy. It is also observed that more efficient jamming is performed when the normalized noise of the jammer is non-Gaussian.

To investigate the optimum power allocation and the on-off jamming strategies for the scenario in Fig. 1, Table I presents the PDFs of the power allocation parameter $\gamma$ for various $\beta$ values in the non-Gaussian jammer scenario. It is observed that the optimum power allocation can result in a randomization between two nonzero power levels, which is different from the on-off jamming approach [4].

Finally, the improvability and the nonimprovability conditions in Proposition 2 and 3 are investigated for the nonGaussian jammer scenario in Fig. 1. Fig. 2 illustrates $\mathrm{F}^{\prime \prime}(\beta)$, which is defined as the second derivative of $\mathrm{P}_{\mathrm{D}}(\sqrt{a})$ at $a=\beta$. As stated in Proposition 2, jamming performance is improved via the optimum power allocation approach whenever $\mathrm{F}^{\prime \prime}(\beta)$ is negative. It is observed from Fig. 1 and Fig. 2 that Proposition 2 presents sufficient but not necessary conditions for improvability since $\mathrm{F}^{\prime \prime}(\beta)$ in Fig. 2 is negative for $\beta \in[0.133,0.268]$ and $\beta \in[0.346,0.633]$ whereas
TABLE I

FOR THE NON-GAUSSIAN JAMMER SCENARIO IN FIG. 1, PDFS FOR OPTIMAL RANDOMIZATION AND ON-OFF JAMMER SPECIFIED BY $p_{\gamma}(\gamma)=\lambda \delta\left(\gamma-\gamma_{1}\right)+(1-\lambda) \delta\left(\gamma-\gamma_{2}\right)$ AND $p_{\gamma}(\gamma)=\lambda_{\text {on }} \delta\left(\gamma-\gamma_{\text {on }}\right)+\left(1-\lambda_{\text {on }}\right) \delta(\gamma)$, RESPECTIVELY

\begin{tabular}{|c||c|c|c||c|c|}
\hline$\beta$ & $\lambda$ & $\gamma_{1}$ & $\gamma_{2}$ & $\lambda_{\text {on }}$ & $\gamma_{\text {on }}$ \\
\hline 0.25 & 0.748 & 0.255 & 0.894 & 1 & 0.5 \\
0.5 & 0.407 & 0.255 & 0.894 & 0.665 & 0.867 \\
0.75 & 0.067 & 0.255 & 0.894 & 1 & 0.866 \\
1 & 1 & 1 & N/A & 1 & 1 \\
1.5 & 1 & 1.225 & N/A & 1 & 1.225 \\
\hline
\end{tabular}

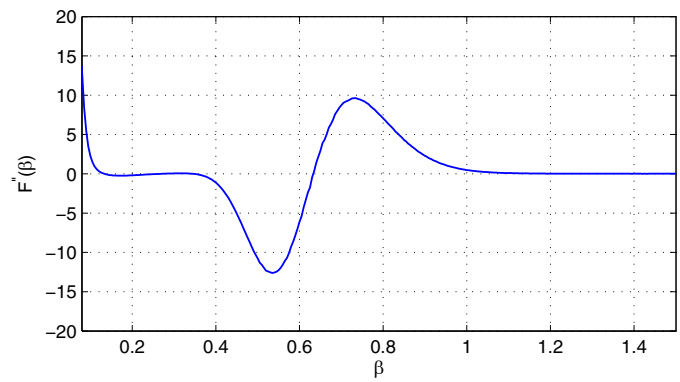

Fig. 2. $\quad F^{\prime \prime}(\beta)$ for the non-Gaussian jammer scenario in Fig. 1 (see Proposition 2).

the improvements are actually achieved for $\beta<0.03$ and $\beta \in[0.06,0.8]$, as can be deduced from a detailed analysis of Fig. 1. When the nonimprovability condition in Proposition 3 is evaluated for various $\beta$ values, it is calculated that the inequality in (10) is satisfied for $\beta \in[0.03,0.06)$ and $\beta>0.8\left(\theta=\mathrm{P}_{\mathrm{D}}^{\prime}(\sqrt{\beta}) /(2 \sqrt{\beta})\right.$ is used as stated in Remark 1$)$. Therefore, in those cases, power randomization cannot provide improvements in jamming performance compared to the no randomization case, which can also be verified from Fig. 1. It is also observed that Proposition 3 provides necessary and sufficient conditions for (non)improvability.

\section{REFERENCES}

[1] C. Goken, S. Gezici, and O. Arikan, "Optimal stochastic signaling for power-constrained binary communications systems," IEEE Trans. Wireless Commun., vol. 9, no. 12, pp. 3650-3661, Dec. 2010.

[2] H. Chen, P. K. Varshney, S. M. Kay, and J. H. Michels, "Theory of the stochastic resonance effect in signal detection-part I: fixed detectors," IEEE Trans. Signal Process., vol. 55, no. 7, pp. 3172-3184, July 2007.

[3] S. Bayram, S. Gezici, and H. V. Poor, "Noise enhanced hypothesistesting in the restricted Bayesian framework," IEEE Trans. Signal Process., vol. 58, no. 8, pp. 3972-3989, Aug. 2010.

[4] M. Azizoglu, "Convexity properties in binary detection problems," IEEE Trans. Inf. Theory, vol. 42, no. 4, pp. 1316-1321, July 1996.

[5] S. Loyka, V. Kostina, and F. Gagnon, "Error rates of the maximumlikelihood detector for arbitrary constellations: convex/concave behavior and applications," IEEE Trans. Inf. Theory, vol. 56, no. 4, pp. 19481960, Apr. 2010.

[6] M. Weiss and S. C. Schwartz, "On optimal minimax jamming and detection of radar signals," IEEE Trans. Aerospace and Electron. Syst., vol. AES-21, no. 3, pp. 385-393, May 1985.

[7] L. H. Zetterberg, "Signal detection under noise interference in a game situation," IRE Trans. Inf. Theory, vol. 8, no. 5, pp. 47-52, Sep. 1962.

[8] I. Krikidis, et. al., "Power allocation for cooperative-based jamming in wireless networks with secrecy constraints," in Proc. 2010 IEEE GLOBECOM Workshops, pp. 1177-1181.

[9] R. J. McEliece and W. E. Stark, "An information theoretic study of communication in the presence of jamming," in Proc. 1981 IEEE International Conference on Communications, pp. 45.3.1-45.3.5.

[10] M. V. Hegde, W. E. Stark, and D. Teneketzis, "On the capacity of channels with unknown interference," IEEE Trans. Inf. Theory, vol. 35, no. 4, pp. 770-783, July 1989.

[11] R. T. Rockafellar, Convex Analysis. Princeton University Press, 1968

[12] F. Moghimi, A. Nasri, and R. Schober, "Adaptive $L_{p}$-norm spectrum sensing for cognitive radio networks," IEEE Trans. Commun., vol. 59, no. 7, pp. 1934-1945, July 2011. 